\title{
Robust and specific inhibition of microRNAs in Caenorhabditis elegans
}

\author{
Samrat T Kundu and Frank J Slack* \\ See research article http://www.silencejournal.com/content/1/1/9
}

\begin{abstract}
MicroRNAs (miRNAs) are small non-coding RNAs that regulate the expression of numerous target genes. Yet, while hundreds of miRNAs have been identified, little is known about their functions. In a recent report published in Silence, Zheng and colleagues demonstrate a technique for robust and specific knockdown of miRNA expression in Caenorhabditis elegans using modified antisense oligonucleotides, which could be utilized as a powerful tool for the study of regulation and function of miRNAs in vivo.
\end{abstract}

MicroRNAs (miRNAs) are approximately 22-nucleotidelong non-coding RNA molecules that were first discovered in Caenorhabditis elegans but are now known to be expressed in all metazoans. miRNA genes are present singly or in clusters, often with their individual promoters and regulatory elements, while some miRNA genes are also present within the introns of other protein coding genes. miRNAs are transcribed as long primary transcripts and are processed first in the nucleus by the microprocessor DRSH-1/DROSHA and then in the cytoplasm by the DCR-1/DICER complex to generate the active 21 to 23-nucleotide RNA molecules. These are recruited by the RNA-induced silencing complex (RISC) and guided to the 3' untranslated regions of target messenger RNAs (mRNAs) with complete or incomplete sequence complementarity, resulting in either degradation of the target mRNA or inhibition of its translation. A single miRNA can target multiple mRNAs and this ability to control the expression of numerous genes makes miRNAs pivotal in regulating various life

*Correspondence: frank.slack@yale.edu

Department of Molecular, Cellular and Development Biology, Yale University, New Haven, CT 06520, USA processes, from development through metabolism to senescence, aging and death (reviewed in [1]).

While animals like $C$. elegans contain hundreds of miRNAs, little is known about their functions. Moreover, with the advent of deep sequencing technology, novel miRNAs are being discovered in different model organisms, requiring faster and more convenient methodologies to study their functional importance through inhibiting their activity in vivo. A recent report by Zheng and colleagues [2] now demonstrates the efficient and specific inhibition of miRNAs in C. elegans by dextran-conjugated modified antisense oligonucleotides.

miRNAs were first identified in C. elegans in 1993 [3] and since then this elegant model system has been extensively utilized for functional analysis of miRNAs. $C$. elegans is so useful for these analyses because of its easy genetics, completely sequenced genome and simple anatomy. To study the role of individual miRNAs in different cellular pathways, forward genetics approaches have yielded many 'loss of function' (lf) mutant strains for various miRNAs [4,5]. Although such mutant strains have been extensively used for functional studies of the target miRNAs, their generation is both time consuming and labor intensive. Further, it is tedious to generate knockout alleles for miRNAs that are essential for survival and development, and in cases where the miRNAs are located in the intronic sequences of protein coding genes, it is possible that their knockout will perturb expression of the protein coding gene. It is also difficult to specifically knock out a single miRNA from a miRNA gene cluster without affecting the expression of the remaining miRNAs in the cluster.

Many groups have tried using reverse genetics approaches to inhibit specific miRNA function transiently in different model systems. The most popular tool of choice is differently modified antisense oligonucleotides, which are easy to synthesize and deliver. Several research groups have demonstrated inhibition of miRNA function with limited success using antisense oligonucleotides such as locked nucleic acids (modified RNA nucleotides) [6] or morpholinos (nucleic 
acid analogs) [7] in Drosophila, zebrafish, and mice [8-10].

C. elegans has been widely used as the model system to study the biological role of small non-coding RNAs and yet, to date, no standard techniques or protocols are available to effectively and conveniently knockdown miRNA function transiently. To inhibit miRNA expression in C. elegans, Zamore's group injected 2'-oxymethyl oligonucleotides into developing embryos [11]. Their embryo injection technique is technically difficult and, therefore, has not been used extensively. Furthermore, the primary drawback of using these modified oligonucleotides is the incumbent toxicity caused by poor solubility and inadequate cytoplasmic retention and tissue distribution.

To address these issues, Zheng and colleagues [2] conjugated 2'-oxy-methyl antisense oligonucleotides complementary to the target miRNAs with the polysaccharide dextran, which has high solubility in water and shows increased cellular uptake and availability. The authors also modified these oligonucleotides by conjugating one to three molecules of rhodamine per molecule of dextran. These modified antisense oligonucleotides were injected into the syncytial gonads of adult worms and embryos were selected based on the presence of rhodamine (Figure 1).

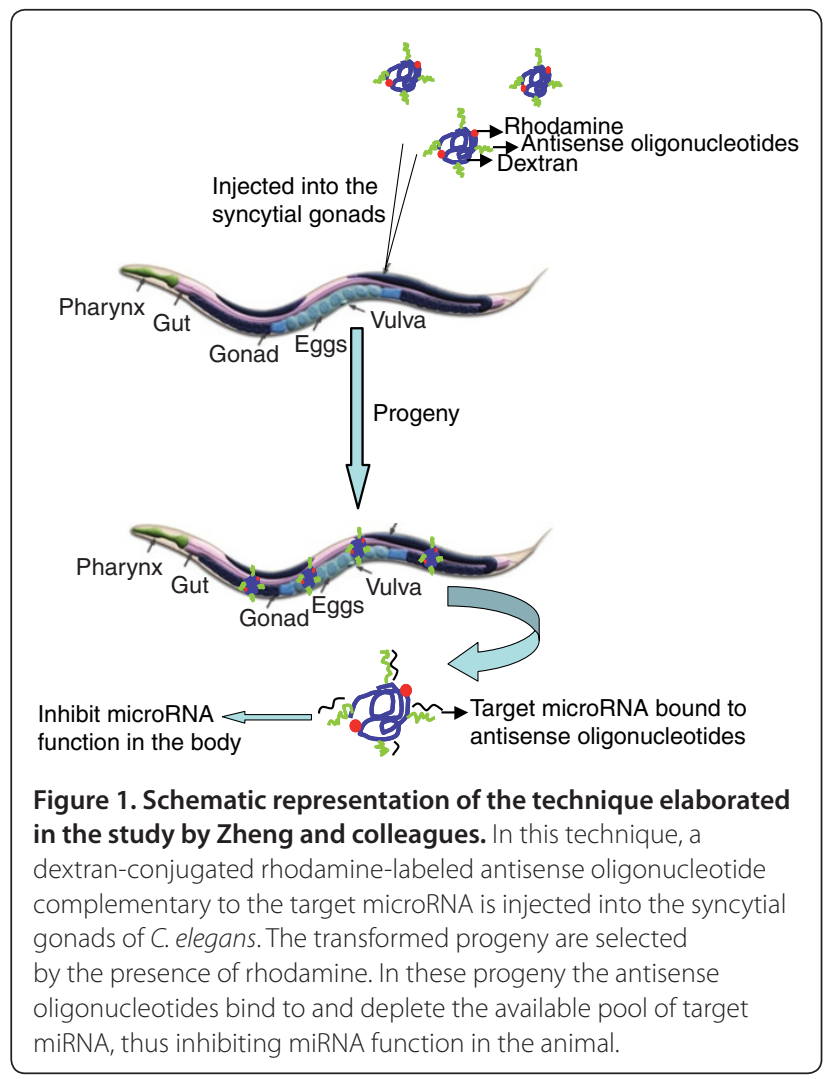

Zheng and colleagues [2] used antisense oligonucleotides complementary to lin-4 and let-7 and comprehensively demonstrated robust knockout phenotypes similar to those seen in the respective loss of function mutant strains of lin-4 and let-7. For example, progeny of animals injected with antisense oligonucleotides against lin-4 show delayed exit in differentiation of seam cells and lack of vulva formation, known phenotypes for lin-4(lf) [3]. The progeny of worms injected with antisense oligonucleotides complementary to let-7 demonstrated a bursting vulva phenotype with lack of alae (longitudinal ridge) formation in $100 \%$ of animals, consistent with the phenotype shown by let-7 knockout mutant animals [5]. By contrast, progeny of animals treated with antisense oligonucleotides complementary to miR-84, a let-7 family member, exhibited no visible phenotype, consistent with the known phenotype of mir-84(lf) mutants. miR-84 has a sequence that is highly homologous to let- 7 sequence, yet the phenotype observed in the miR-84 knockdown animals was completely unlike the let-7 knockdown phenotype therefore confirming the specificity of inhibition. Using a similar strategy, the authors could demonstrate specific inhibition of lys-6 miRNA in the ASEL neurons, where it functions to maintain left-right symmetry. This technique also successfully demonstrated the inhibition in activity of miR-42, which is active only during embryonic development. In addition, the authors demonstrate that this new class of antisense oligonucleotides could be utilized for specific and simultaneous inhibition of two miRNAs, in this case $l i n-4$ and lys-6.

As with all antisense techniques, the dextranconjugated antisense 2'-oxy-methyl oligonucleotides have a limitation, in this case with respect to the period of efficacy of inhibition against target miRNAs in the animal. The inhibition was robust until 15-20 hours after the L4 molt with higher doses of injection, after which the effect diminished, most probably due to the dilution of the antisense oligonucleotides inside the animal. For this reason this method of miRNA inhibition might not be the most effective for long-term functional assays, such as aging assays, where the miRNA in question needs to be inhibited throughout the life span of the animal.

Overall, the data presented in the report by Zheng and colleagues [2] convincingly suggest that this new class of antisense oligonucleotides could prove to be a very powerful and effective tool for the robust and specific inhibition of miRNA function in vivo. This technique would be very useful for studying the different biological functions of essential and novel miRNAs in C. elegans. While it remains to be tested, this technology does not appear to be limited to just C. elegans, and could be applicable in other organisms as well. 


\section{Published: 1 April 2010}

\section{References}

1. Esquela-Kerscher A, Slack FJ: Oncomirs - microRNAs with a role in cancer. Nat Rev Cancer 2006, 6:259-269.

2. Zheng G, Ambros V, Li W: g miRNA in Caenorhabditis elegans using a potent and selective antisense reagent. Silence 2010, 1:9

3. Lee RC, Feinbaum RL, Ambros V: The C. elegans heterochronic gene lin-4 encodes small RNAs with antisense complementarity to lin-14. Cell 1993, 75:843-854

4. Miska EA, Alvarez-Saavedra E, Abbott AL, Lau NC, Hellman AB, McGonagle SM, Bartel DP, Ambros VR, Horvitz HR: Most Caenorhabditis elegans microRNAs are individually not essential for development or viability. PLOS Genet 2007, 3:e215.

5. Reinhart BJ, Slack FJ, Basson M, Pasquinelli AE, Bettinger JC, Rougvie AE, Horvitz HR, Ruvkun G: The 21-nucleotide let-7 RNA regulates developmental timing in Caenorhabditis elegans. Nature 2000, 403:901-906.

6. Orom UA, Kauppinen S, Lund AH: LNA-modified oligonucleotides mediate specific inhibition of microRNA function. Gene 2006, 372:137-141.
7. Flynt AS, Li N, Thatcher EJ, Solnica-Krezel L, Patton JG: Zebrafish miR-214 modulates Hedgehog signaling to specify muscle cell fate. Nat Genet 2007, 39:259-263.

8. Boutla A, Delidakis C, Tabler M: Developmental defects by antisensemediated inactivation of micro-RNAs 2 and 13 in Drosophila and the identification of putative target genes. Nucleic Acids Res 2003, 31:4973-4980.

9. Krützfeldt J, Rajewsky N, Braich R, Rajeev KG, TuschI T, Manoharan M, Stoffel M: Silencing of microRNAs in vivo with 'antagomirs'. Nature 2005, 438:685-689.

10. Esau C, Kang X, Peralta E, Hanson E, Marcusson EG, Ravichandran LV, Sun Y, Koo S, Perera RJ, Jain R, Dean NM, Freier SM, Bennett CF, Lollo B, Griffey R: MicroRNA-143 regulates adipocyte differentiation. J Biol Chem 2004, 279:52361-52365.

11. Hutvágner G, Simard MJ, Mello CC, Zamore PD: Sequence-specific inhibition of small RNA function. PLoS Bio/ 2004, 2:e98.

doi:10.1186/jbiol230

Cite this article as: Kundu ST and Slack FJ: Robust and specific inhibition of microRNAs in Caenorhabditis elegans. Journal of Biology 2010, 9:20 